\section{An interacting-item scale construction package}

\section{JAMES S. ROBERTS and ROBERT J. JANNARONE University of South Carolina, Columbia, South Carolina}

The SAS ${ }^{\circledast}$ macroprogram and associated subprograms described here provide a new approach to scale construction that reflects interacting items through cross-product scores. Jannarone and Roberts (1984) described a method for binary tests that generates cross-product items in order to increase reliability. The method is unique in that it allows for the identification of potentially useful, nonadditive information, without the use of an external criterion. The SAS macroprogram COMBINE and its associated subprograms perform the necessary steps and evaluate the resulting augmented scale. COMBINE can be used with any set of binary-coded items in order to develop augmented scales. It can also be used with graded response (e.g., Likert) items if they are first dichotomized so that interesting, nonadditive behavior may be uncovered. However, the method may incorrectly identify appropriate cross-product items if the items in question have either very high or low passing proportions. For this reason, caution should be used when examining binary items that have unusual frequency distributions.

Method. The method involves an exploratory and a confirmatory stage. In the exploratory stage, the program first conducts an ANOVA for each pair of binary (passfail) items in an n-item test. For each test, the criterion variable is the subject's number-correct score on the remaining $\mathrm{n}-2$ items, and each subject is placed in one of four cells based on scores for the two items. A $2 \times 2$ factorial ANOVA then yields two item main effects and an interaction effect. For each item pair, COMBINE attempts to fill each cell in the factorial design with a prespecified equal number of subjects. However, when there are too few responses to fill the cells, the analysis is based on the largest possible equal number of subjects in each cell. The approach is formally identical to regressing an individual's total score, minus the scores of the two items being evaluated, on each of the item scores as well as their cross-product (Jannarone \& Roberts, 1984). After all ANOVAs are performed at the user-specified critical level, the number of interactive pairs is accessed. If the number of interactive pairs exceeds expected chance levels, the analysis continues.

The macroprogram then creates binary cross-product items for each of the most interactive item pairs, not to exceed $n / 2$, generating and subsequently executing the

Program development was sponsored by the Social and Behavioral Sciences Laboratory at the University of South Carolina. J. S. Roberts's mailing address is: Social and Behavioral Sciences Laboratory, Gambrell Hall, University of South Carolina, Columbia, SC 29208. necessary SAS code. (For a description of cross-product item scoring, see Jannarone \& Roberts, 1984.) Next, augmented scales are formed for each subject by adding each cross-product score to the number-correct score. The final step in the exploratory stage involves assessing the difference in split-half correlations between the numbercorrect and augmented scales, using a standard test (Olkin, 1967).

In the confirmatory stage, the previously developed item cross-product scores are computed for a new group of subjects. Split-half correlations are again computed on both the original and augmented scales and their difference is assessed.

Jannarone and Roberts (1984) analyzed the 18 California Personality Inventory (CPI) subscales using this method. Of the 12 subscales in which the number of interactive item pairs exceeded chance level, all 12 yielded augmented subscales with split-half correlations that were larger than the originals. In all but three cases, these reliability differences were significant. An interactive test with improved reliability also has been constructed for a verbal analogies test.

Input. Input includes a collection of binary item scores as well as the SAS DATA, INPUT, and CARDS statements that describe the exploratory data set (and the confirmatory data set if requested). Input also includes the number of original scale items, the maximum equal cell size and significance level to be used in the ANOVA phase, and the name of the exploratory data set.

Output. Output from COMBINE includes the ratio of interactive pairs found relative to that expected by chance, the SAS code which is generated to produce augmenting items, and an ANOVA summary table for each interactive item pair. Descriptive statistics and correlations for both the original and augmented split-scales are also provided, as well as a test for their difference.

Language and Development. The macroprogram is written in OS SAS and will run on release 82.3 and 82.4. The program has been tested on an Amdahl 470-V6 mainframe.

Availability. A listing of the program and associated documentation are available at no cost from J. S. Roberts. Requests for tape copies should include a $\$ 20.00$ check or money order to cover costs.

\section{REFERENCES}

JANNARONE, R. J., \& RoBeRTs, J. S. (1984). Reflecting interactions among personality items: Meehl's paradox revisited. Journal of Personality and Social Psychology, 47, 621-628.

Olkin, I. (1967). Correlations revisited, in J. Stanley (Ed.), Improving Experimental Design and Statistical Analysis (pp. 102-108). Chicago: Rand McNally.

(Revision accepted for publication April 26, 1985.) 